\title{
Dynamical evolution of the Universe in the quark-hadron phase transition and possible nugget formation
}

\author{
Deepak Chandra \\ Physics Department, S. G. T. B. Khalsa College, \\ University of Delhi, Delhi-11000\%, India. \\ Ashok Goyal 1 \\ Department of Physics and Astrophysics, \\ University of Delhi, Delhi-11000\%, India. \\ and Inter University Center for Astronomy and Astrophysics, \\ Ganeshkhind, Pune-41100\%, India.
}

\begin{abstract}
We study the dynamics of first-order phase transition in the early Universe when it was $10-50 \mu \mathrm{s}$ old with quarks and gluons condensing into hadrons. We look at how the Universe evolved through the phase transition in small as well as large super cooling scenario, specifically exploring the formation of quark nuggets and their possible survival. The nucleation of the hadron phase introduces new distance scales in the Universe, which we estimate along with the hadron fraction, temperature, nucleation time etc. It is of interest to explore whether there is a relic signature of this transition in the form of quark nuggets which might be identified with the recently observed dark objects in our galactic halo and account for the Dark Matter in the Universe at present.
\end{abstract}

${ }^{1} \mathrm{E}-$ mail:agoyal@ducos.ernet.in 
It is well known that a phase transition from quark gluon plasma to confined hadronic matter must have occurred at some point in the evolution of the early Universe, typically at around $10-50 \mu s$ after the Big Bang. This leads to an exciting possibility of the formation of quark nuggets through the cosmic separation of phases [1]. As the temperature of the Universe falls below the the critical temperature $T_{c}$ of the phase transition, the quark gluon plasma super cools and the transition proceeds through the bubble nucleation of the hadron phase. The typical distance between the nucleated bubbles introduces a new distance scale to the Universe which depends critically on the super cooling that takes place. This bubble nucleation of the hadron phase is governed by the formation of critical bubbles, that is bubbles bigger than the critical size start to expand and coalesce till the entire Universe is filled with the hadron phase. As the hadronic bubbles expand, they heat the surrounding plasma, shutting off further nucleation and the two phases coexist in thermal equilibrium. The hadron phase expands driving the deconfined quark phase into small regions of space and it may happen that the process stops after the quarks reach sufficiently high density to provide enough pressure to balance the surface tension and the pressure of the hadron phase. The quark matter trapped in these regions constitute the quark nuggets. The number of particles trapped in the quark nugget, its size and formation time are dependent sensitively on the degree of super cooling. The duration of the phase transition also depends on the expansion of the Universe and on other parameters like the bag pressure $B$ and the surface tension $\sigma$.

The quark nuggets formed in the small super cooling scenario are in a hot environment around the critical temperature $T_{c}$ and are susceptible to evaporation from the surface [2] and to boiling through subsequent hadronic bubble nucleation inside the nuggets [3]. However in the large super cooling scenario we have the interesting possibility of these nuggets forming at a much lower temperature than $T_{c}$ due to the long time of phase transition and consequent expansion of the Universe. Alcock and Farhi [2] have shown that the quark nuggets with baryon numbers $\leq 10^{52}$ and mass $\leq 10^{-5} M_{\odot}$ are unlikely to survive evapora- 
tion of hadrons from the surface. Boiling was shown to be even more efficient mechanism of nugget destruction by Alcock and Olinto [3]. If these nuggets are formed at around $100 \mathrm{MeV}$, they cannot have more baryons than are allowed in the horizon by the standard model of Cosmology, i.e. $\sim 10^{49}\left(\frac{T}{100 \mathrm{MeV}}\right)^{-3}$ and would not survive till the present epoch. We will see that for small super cooling this is the likely fate of the nuggets. These results were somewhat modified by Madsen et.al. 顿 by taking into account the flavor equilibrium near the nugget surface for the case of evaporation and by considering the effect of interactions in the hadronic gas in a relativistic mean field model described by Walecka for the case of boiling [5]. They suggested that the quark nuggets with large baryon number allowed by the causality limit may after all be able to survive from the early Universe. In the large super cooling scenario the time at which these nuggets are formed can be quite late along with a much lower formation temperature due to the expansion of the Universe. Such nuggets can have a baryon number content of $\geq 10^{52}$ and being at a much lower temperature than $T_{c}\left(\leq 0.1 T_{c}\right)$ will easily survive till the present epoch. The number of baryons in the horizon (of size $\sim 2 t$ ) can also be large due to long time it takes for the bubbles to meet and form the nuggets.

There have been recent observations by gravitational micro lensing [6] of dark objects in our galactic halo having masses of about $0.01-1$ solar mass. If these objects have to be identified with quark nuggets, they could only have been formed at a time later than the time when the Universe cooled through $T_{c}$, i.e. later than 50-100 $\mu$ s after the Big Bang. Such a possibility of the nuggets forming at a temperature $\sim 0.1 \mathrm{MeV}$, implying a high degree of super cooling and strongly first order phase transition was recently investigated by Cottingham et. al. [7] in the Lee-Wick model [8]. Their investigation showed that the time of formation and the baryon content of these nuggets are essentially determined by the rate at which the hadron bubbles nucleate. However there was still the question of reheating due to the expansion of the bubbles of hadron phase which raises the temperature towards $T_{c}$. These studies have also been carried out by taking interactions into account in both the phases and by incorporating 
the effects of curvature [9] energy in the calculations.

Studies of quark-hadron phase transitions in the early Universe [10], in heavy ion collision 11] and in high density nuclear matter have been done previously by looking in detail at the dynamics of the phase transition. Kapusta et. al. have applied a recently computed nucleation rate [1] to a first order phase transition in a set of rate equations to study the time evolution of a quark-gluon plasma as it converts to hadronic matter in heavy ion collisions. Based on Bjorken hydrodynamics and on current parameter values, they find the transition generates 30 percent extra entropy and also a time delay of $\sim 11 \mathrm{fm} / \mathrm{c}$ in completion of the transition. Kajantie and Kurki-Suonio [10] studied how the early Universe during the quark-hadron phase transition evolved through the mixed phase in a scenario with small initial super cooling. Fuller et.al. [10] have also studied the dynamics of the Universe during the constant temperature coexistence epoch.

In this letter we calculate the nucleation rate and quantitatively study what happens when the temperature drops to $T_{c}$. This nucleation rate is used to solve a set of rate equations to study the time evolution of the quark-gluon phase as it converts to hadronic matter in an expanding Universe. A novel feature of this method is that reheating of the plasma during phase transition is included in the calculations and all relevant quantities can be evaluated as a function of time.

Bubble Nucleation:- When the early Universe as a quark-gluon thermodynamic system cools through the critical temperature $T_{c}$, energetically, the new phase remains unfavorable as there is free energy associated with surface of separation between the phases. Small volumes of new phase are thus unfavorable and all nucleated bubbles with radii less than critical radius collapse and die out. But those with radii greater than the critical radius expand until they coalesce with each other. So super cooling occurs before the new phase actually appears and is then followed by reheating due to release of latent heat. The bubble nucleation rate [10] at temperature $\mathrm{T}$ is given by

$$
I=I_{o} e^{-\frac{W_{c}}{T}}
$$


where $I_{o}$ is the prefactor having dimension of $T^{4}$. The prefactor used traditionally in early Universe studies [10 is given by $I_{o}=\left(\frac{W_{c}}{2 \pi T}\right)^{\frac{3}{2}} T^{4}$. Csernai and Kapusta 11] have recently computed this prefactor in a coarse-grained effective field theory approximation to QCD and give $I_{o}=\frac{16}{3 \pi}\left(\frac{\sigma}{3 T}\right)^{\frac{3}{2}} \frac{\sigma \eta_{q} R_{c}}{\xi_{q}^{4}(\Delta w)^{2}}$ where $\eta_{q}=14.4 T^{3}$ is the shear viscosity in the plasma phase, $\xi_{q}$ is a correlation length of order $0.7 \mathrm{fm}$ in the plasma phase and $\Delta w$ is the difference in the enthalpy densities of the two phases. In this letter we use both these prefactors for comparison. The critical bubble radius $R_{c}$ and the critical free energy $W_{c}$ are obtained by maximizing the thermodynamic work expended to create a bubble and are given by $R_{c}=\frac{2 \sigma}{P_{h}(T)-P_{q}(T)}$ and $W_{c}=4 \pi \sigma R_{c}^{2}(T) / 3=\frac{16 \pi \sigma^{3}}{3 \Delta P^{2}}$ where $\Delta P=P_{h}(T)-P_{q}(T)$ is the pressure difference in hadron and quark phase.

For simplicity we describe the quark matter by a plasma of massless $u, d$ quarks and massless gluons without interaction. The long range non-perturbative effects are parameterized by the bag constant $B$. The light particles (photons, neutrinos, and electrons) contribute equally to the pressure in both the phases. The pressure in the QGP phase is given by $P_{q}(T)=\frac{1}{3} g_{q} \frac{\pi^{2}}{30} T^{4}-B$ where $g_{q} \sim 51.25$ is the effective number of degrees of freedom. In the hadronic phase the pressure is given by $P_{h}(T)=\frac{1}{3} g_{h} \frac{\pi^{2}}{30} T^{4}$ where $g_{h} \sim 17.25$, taking the three pions as massless.

The fraction of the Universe $h(t)$ which has been converted from QCD plasma phase to hadronic phase at the time $t$ was first given by Guth and Weinberg [12] and applied to cosmological first order phase transitions. Csernai and Kapusta [11] gave a kinetic equation for calculating $h(t)$ which takes bubble growth into account. If the early Universe cools to $T_{c}$ at time $t_{c}$, then at same later time $t$ the fraction of the Universe in hadronic phase is given by the kinetic equation

$$
h(t)=\int_{t_{c}}^{t} I\left(T\left(t^{\prime}\right)\right)\left[1-h\left(t^{\prime}\right)\right] V\left(t^{\prime}, t\right)\left[\frac{R\left(t^{\prime}\right)}{R(t)}\right]^{3} d t^{\prime}
$$

where $V\left(t^{\prime}, t\right)$ is the volume of a bubble at time $t$ which was nucleated at an earlier time $t^{\prime}$ and $R(t)$ is the scale factor. This takes bubble growth into account 
and can be given simply as

$$
V\left(t^{\prime}, t\right)=\frac{4 \pi}{3}\left[R_{c}\left(T\left(t^{\prime}\right)\right)+\int_{t^{\prime}}^{t} \frac{R(t)}{R\left(t^{\prime \prime}\right)} v\left(T\left(t^{\prime \prime}\right)\right) d t^{\prime \prime}\right]^{3}
$$

where $v(T)$ is the speed of the growing bubble wall and can be taken to be $v(T)=v_{o}\left[1-\frac{T}{T_{c}}\right]^{\frac{3}{2}}$ where $v_{o}=3 c$. This has the correct behavior in that closer $T$ is to $T_{c}$ slower do the bubbles grow. When $T=\frac{2}{3} T_{c}$ we have $v(T)=\frac{1}{\sqrt{3}}$ the speed of sound of a massless gas. For $T<\frac{2}{3} T_{c}$ which occurs when there is large super cooling, we use the value $v(T)=\frac{1}{\sqrt{3}}$. In this analysis collision and fusion of bubbles have not been taken into consideration. This seems to be justified as far as fusion of bubbles is concerned. Witten [1] and Kurki Suonio [10] have shown that for small enough bubbles, surface tension will cause them to coalesce into larger bubbles. This distance scale has been estimated to be given by $l_{c}=3\left(\frac{\sigma}{T_{c}^{3}}\right)^{\frac{1}{3}}\left(\frac{T_{c}}{200 M e V}\right)^{-\frac{5}{3}} \mathrm{~mm}$. If nucleation scale $l_{n}$ is larger than $l_{c}$ then we are justified in neglecting fusion of bubbles. As we will see, this is the case in all interesting scenarios.

The other equation we need is the dynamical equation which couples time evolution of temperature to the hadron fraction $h(t)$. We use the two Einstein's equations as applied to the early Universe neglecting curvature.

$$
\begin{aligned}
& \frac{\dot{R}}{R}=\sqrt{\frac{8 \pi G}{3}} \rho^{\frac{1}{2}} \\
& \frac{\dot{R}}{R}=-\frac{1}{3 w} \frac{d \rho}{d t}
\end{aligned}
$$

where $w=\rho+P$ is the enthalpy density of the Universe at time $t$. The energy density in the mixed phase is given by $\rho(T)=h(t) \rho_{h}(T)+[1-h(t)] \rho_{q}(T)$, where $\rho_{h}$ and $\rho_{q}$ are the energy densities in the two phases at temperature $T$ and similarly for enthalpy. We have numerically integrated the coupled dynamical equations (2),(4) and(5) to study the evolution of the phase transition starting above $T_{c}$ at some temperature $T$ corresponding to time $t$. This time $t$ has been obtained by integrating the Einstein's equation (4) and (5) and is given by

$$
t=-\sqrt{\frac{9}{24 \pi G}} \int_{\infty}^{T} \frac{d T}{\left(\frac{g_{q} \pi^{2} T^{6}}{30}+B T^{2}\right)^{1 / 2}}
$$


The number density of nucleated sites at time $t$, is given by

$$
N(t)=\int_{t_{c}}^{t} I\left(t^{\prime}\right)\left[1-h\left(t^{\prime}\right)\right]\left(\frac{R\left(t^{\prime}\right)}{R(t)}\right)^{3} d t^{\prime}
$$

Therefore the typical separation between nucleation sites is $l_{n}=N(t)^{-1 / 3}$. This distance scale will eventually determine the number of quarks in a nugget. This scale can be up to $10^{12} \mathrm{Km}$ depending on the parameters $B$ and $\sigma$ which correspond to a distance of $\sim 1.4 M p c$ today. Thus the structures generated in this transition can be of the order of distance between galaxies today. The observable separation of galaxies in the Universe can be a remnant of this transition with the centers of the galaxies being the quark nuggets. Of course the collision of bubbles and their random nucleation and interaction will also lead to clustering of the nuggets, which can qualitatively explain the clustering of galaxies.

To get an idea about the super cooling before nucleation begins, we can plot the nucleation time as a function of temperature, defined by $\tau_{\text {nucleation }}^{-1}=\frac{4 \pi R_{c}^{3}}{3} I$. This characteristic time scale neglects bubble growth. The quark number density in the early Universe is given by $n_{q}=\frac{2}{\pi^{2}} \zeta(3)\left(\frac{n_{q}}{n_{\gamma}}\right) T^{3}$ where $\frac{n_{q}}{n_{\gamma}}$ is the quark to photon ratio estimated from the abundance of luminous matter in the Universe to be roughly equal to $3 \times 10^{-10}$. The quark nuggets which have trapped the quarks in the plasma phase have $N_{q}$ quarks at time $t$ given by the number of quarks in a volume $\frac{1}{N(t)}$, i.e. $N_{q}=\frac{n_{q}}{N(t)}$. The nucleation sites are actually randomly distributed, but we expect a distribution of quark numbers around $N_{q}$. The average temperature at which nuggets are formed when bubbles coalesce is obtained by finding the average time at which the expanding bubble surfaces meet. Assuming a cubic lattice, this is given by setting twice the radius of the expanding bubbles equal to the length scale $l_{n}$ of the lattice spacing. We have done this numerically to get the corresponding time $t_{f}$ and temperature $T_{f}$ and these quantities have been estimated for different values of $B$ and $\sigma$. When the fraction of the space occupied by the bubbles is around 50 percent, we expect the bubbles to meet in an ideal picture,i.e. if all bubbles are essentially nucleated at one instant which is the maximum nucleation time and they all have the same radius. However we have a distribution of expanding bubble sizes because 
of the different points of time at which they were nucleated. Therefore the estimate of the time of nugget formation by treating all bubbles to be of the same size is an underestimate. We find that hadron fraction $h(t)$ is only around .12 when bubbles meet by this criteria. However we do not expect this to change qualitatively the broad picture of the transition and the nugget formation apart from reducing the formation time.

In Fig. 1 we have plotted the $\log _{10}$ of nucleation time $\tau$ as a function of temperature for different values of the bag pressure $B$, surface tension $\sigma$ and the prefactor $I_{o}$. These curves clearly show when nucleation becomes large and how much super cooling of the Universe occurs. Fig.2 shows the temperature as a function of time. It is clear from this diagram that reheating takes place as nucleation begins. As $\sigma$ increases, the super cooling is larger and the transition takes much longer to complete with more chance of nugget formation. At the bottom of the curves, nucleation starts with release of latent heat and consequent rise in temperature. When the transition completes, the Universe again starts cooling. However, larger the super cooling, slower is the reheating as the Universe keeps on expanding. This allows the nuggets to be formed at a much lower temperature when bubble walls meet. From Fig.3 we see that the average bubble density $N(t)$ is initially zero and then increases with time. As soon as reheating starts, bubble nucleation shuts off at a particular point. The transition now continues only by expansion of the nucleated bubbles. The fall in $N(t)$ beyond this point is due to the expansion of the Universe. Fig.4 shows the fraction $h(t)$ of the Universe in hadron phase as a function of time. For small values of $\sigma$ the transition completes quickly as $h(t)$ goes to 1 . But for larger $\sigma$ it takes a larger time for $h(t)$ to become 1. It may be comparable and even larger than the expansion time scale of the Universe. Thus the dilution factor is very important here as it makes the reheating much slower and consequently delays the transition. We also notice that in the large super cooling scenario the Kapusta prefactor becomes much bigger than the standard one by many orders of magnitude. This makes the nucleation rate as well as the reheating faster.In the case of low super cooling the two prefactors give al- 
most identical results. The number of quarks in the horizon $N_{q H}$ at time $t$ is $N_{q H} \sim n_{q}\left(\frac{4}{3} \pi t^{3}\right) \sim\left(\frac{n_{q}}{n_{\gamma}}\right) \frac{2 \zeta(3)}{3 \pi^{2}} T^{3} 4 \pi t^{3}$ and we find that for all interesting cases $N_{q} \leq N_{q H}$ and this number is very sensitively dependent on the surface tension. Physically it is possible to have $N_{q H} \geq N_{q} \geq 10^{52}$ for some values of the parameters B and $\sigma$. In table I below we list some physical quantities for some representative values of $B$ and $\sigma$.

\begin{tabular}{cccccccc}
\hline$B^{1 / 4}$ & $\sigma$ & $T_{c}$ & $t_{f}$ & $T_{f}$ & $N_{q}$ & $N_{q H}$ & $l_{n}$ \\
$\mathrm{MeV}$ & $\mathrm{MeVfm}^{-2}$ & $\mathrm{MeV}$ & $\mu s$ & $\mathrm{MeV}$ & & & $m$ \\
\hline 235 & 50 & 169 & 12.1 & 169 & $2.6 \times 10^{28}$ & $7 \times 10^{52}$ & $8 \times 10^{-3}$ \\
100 & 39.5 & 71.9 & 2595 & 13.9 & $1.2 \times 10^{50}$ & $3.8 \times 10^{56}$ & $8.4 \times 10^{5}$ \\
113 & 57.1 & 81.3 & 5138 & 12.3 & $9 \times 10^{49}$ & $2 \times 10^{57}$ & $1.7 \times 10^{6}$ \\
\hline
\end{tabular}

Table I. Some relevant physical quantities for some representative values of $B$ and $\sigma$ using the standard prefactor.

As nuggets with $N_{q} \geq 10^{52}$ are expected to survive the transition, they will contribute to the energy density of the universe. However, nuggets of solar mass range need somewhat unrealistically large surface tension, specially if the bag pressure $B$ is also large.

In conclusion, detailed dynamics of the quark-hadron transition in the early Universe show that the evolution of the Universe does not necessarily follow the small super cooling scenario and certain choices of B and $\sigma$ can have a bearing on the present state of the Universe. We have explored in detail the possibility of nugget formation and also estimated their average separation, time of formation, quark content and survivability by solving a rate equation coupled to the Einstein's equations in an expanding universe. Clearly, the analysis can be improved by taking interactions into account in both the phases and also bubble interactions may be incorporated in the calculations. This will be reported elsewhere. however we believe that qualitatively the results given here will hold. If the nuggets studied above are indeed formed in a much cooler environment, they could contribute significantly to the missing mass in the Universe and be candidates for dark matter. 
Acknowledgement: We would like to thank J. V. Narlikar for providing hospitality at the Inter University center for Astronomy and Astrophysics, Pune, India where a part of this work was completed. 


\section{References}

[1] E. Witten, Phys. Rev. D30, 272 (1984); A. Applegate and C.J. Hogan, Phys. Rev. D31, 3037 (1985); E. Farhi and R.L. Jaffe, Phys. Rev. D30, 2379 (1984).

[2] C. Alcock and E. Farhi, Phys. Rev. D32, 1273 (1985).

[3] C. Alcock and A. Olinto, Phys. Rev. D39, 1233 (1989); J.A. Frieman et.el., Phys. Rev. D40, 3241 (1989).

[4] J. Madsen et.al., Phys. Rev. D34, 2947 (1986).

[5] J. Madsen and M.L. Olesen, Phys. Rev. D43, 1069 (1991).

[6] C. Alcock et.al., Nature 365, 621 (1993); E. Aubourg et.al., Nature 365, 623 (1993).

[7] W.N. Cottingham and Vinh Mau, Phys. Rev. Lett. 73, 1328 (1994).

[8] T.D. Lee and G.C. Wick, Phys. Rev. D9, 2291 (1974).

[9] I. Madsor and B. Svetitsky, Phys. Rev. D44, 878 (1991); Ashok Goyal and Deepak Chandra, Astron. Astrophys. 330, 10 (1998).

[10] C.J. Hogan, Phys. Lett. B133, 172 (1983); K. Kajantie and M. KurkiSuonio, Phys. Rev. D34, 1719 (1996); G.M. Fuller,G.J. Mathews and C.R. Alcock, Phys. Rev. D37, 1380 (1988).

[11] L.P. Csernai and J.I. Kapusta, Phys. Rev. Lett. 69, 737 (1992); Phys. Rev. D46, 1379 (1992).

[12] A.H. Guth and E.J. Weinberg, Phys. Rev. D23, 376 (1981). 


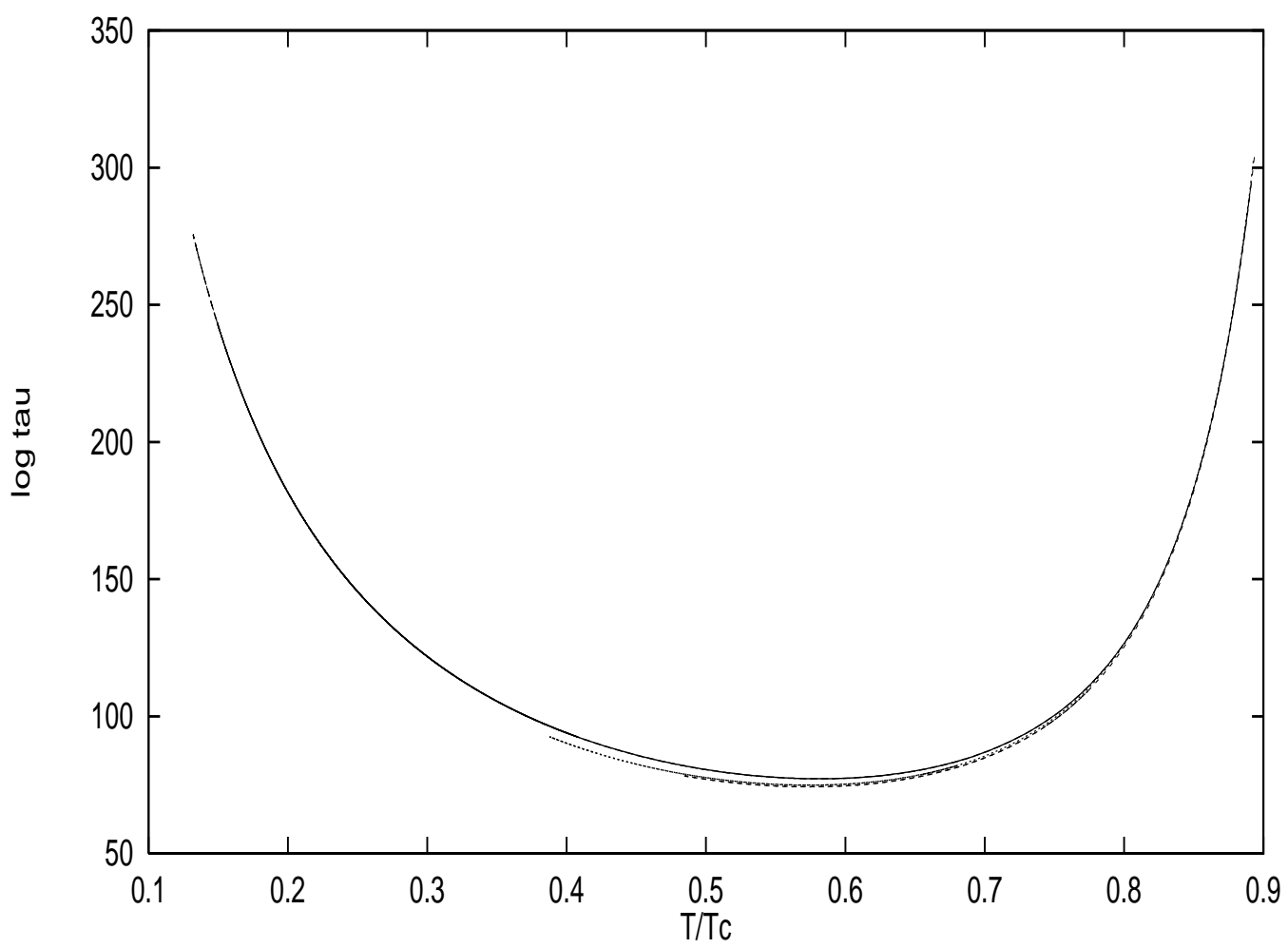

Figure 1: $\log$ of the nucleation time $\tau$ in units of $\mathrm{fm} / \mathrm{c}$ as a function of temperature. Solid and dashed curves are for $B^{1 / 4}=100 \mathrm{MeV}$ and $\sigma=39.5 \mathrm{MeV} \mathrm{fm}^{-2}$ with the standard and the Kapusta prefactors respectively. Long dashed and dotted curves are for $B^{1 / 4}=113 \mathrm{MeV}$ and $\sigma=57.1 \mathrm{MeV} \mathrm{fm}^{-2}$ with the standard and the Kapusta prefactors respectively. 


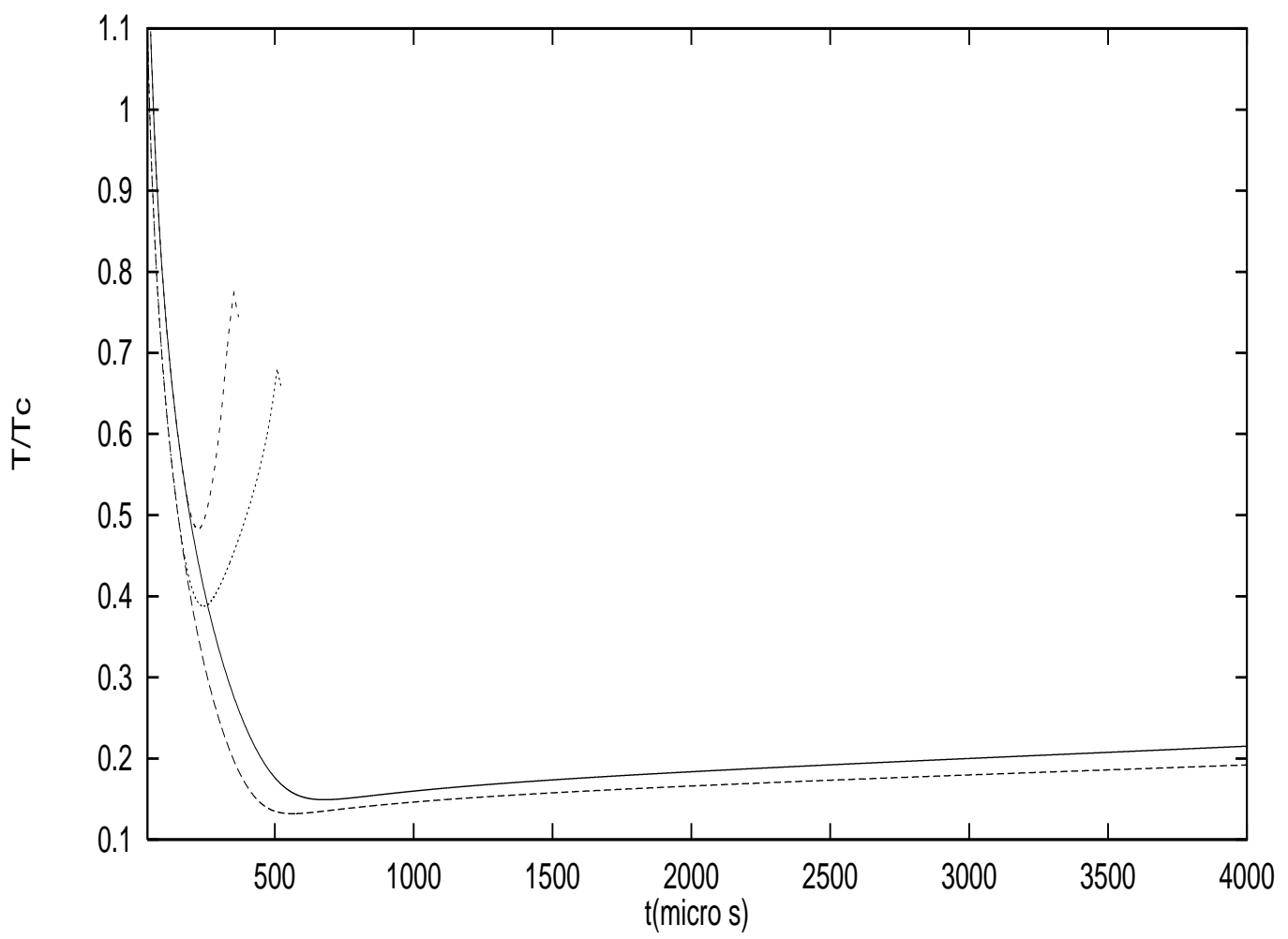

Figure 2: Temperature as a function of time. Solid, dotted, dashed and long dashed curves are as in fig. 1 . 


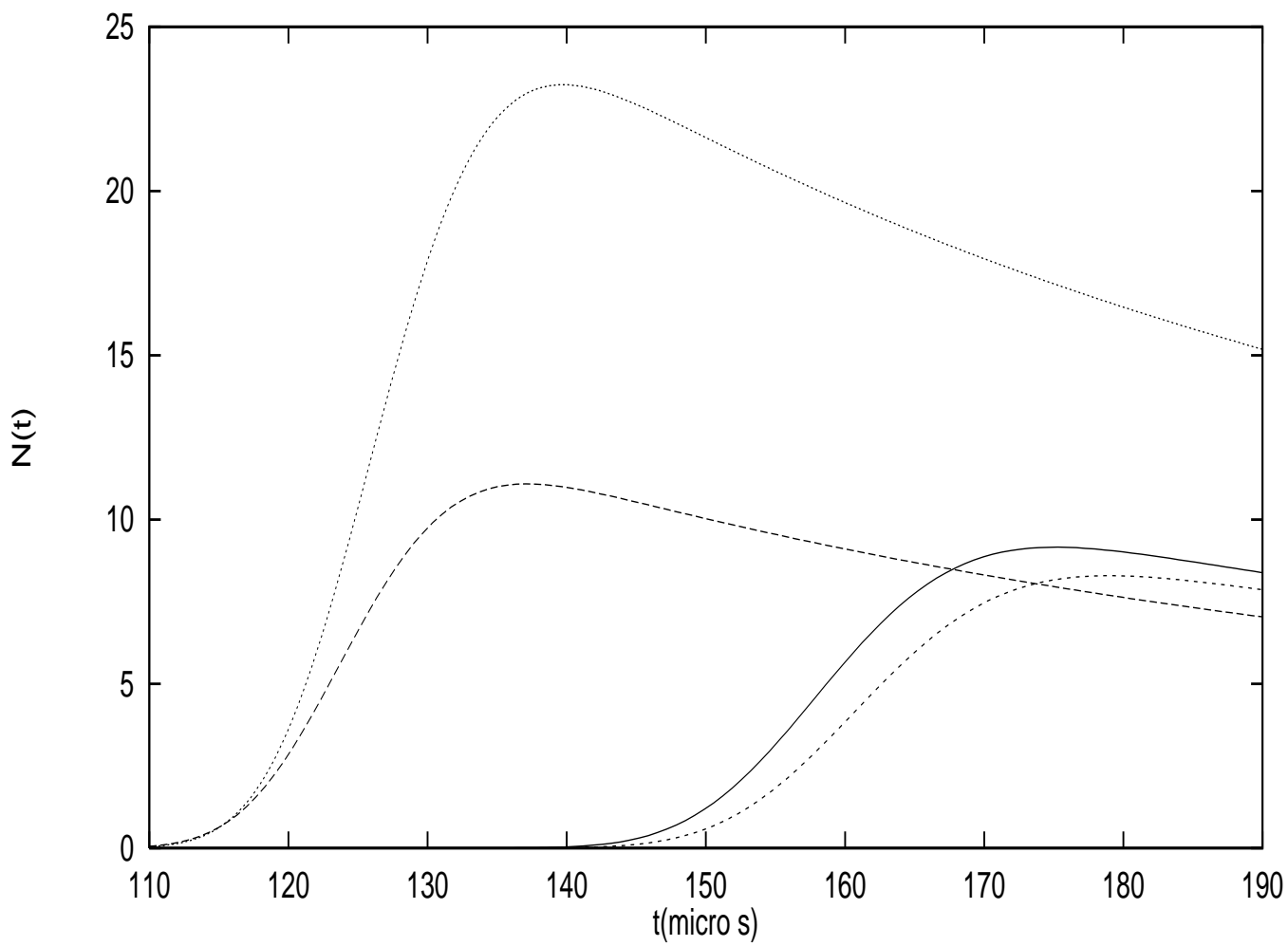

Figure 3: Average bubble density $N(t)$ as a function of time in units of $\left(10^{5} \mathrm{~km}^{3}\right)^{-1}$. The bubble density for the standard prefactor (solid and long dashed curves) is normalized by multiplying with a factor of $10^{3}$. Curves as in fig. 1. 


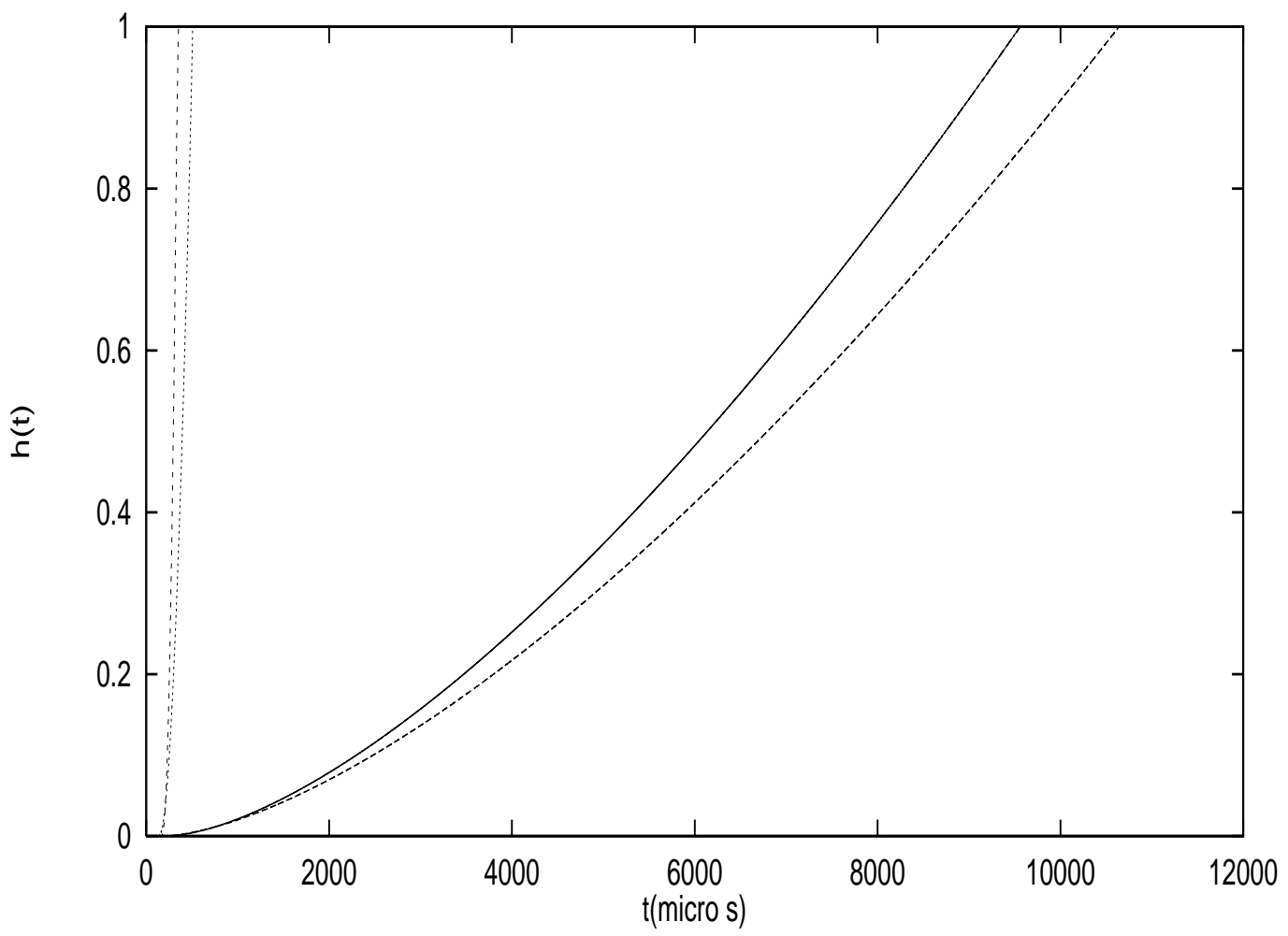

Figure 4: The hadron fraction as a function of time. Curves as in fig. 1. 